\title{
Improving understanding of river corridor ecosystems through integration of multiscale models and observations
}

\author{
T.D. SCHEIBE ${ }^{1 *}$ AND THE PNNL SBR SFA TEAM ${ }^{2}$
}

${ }^{1}$ Pacific Northwest National Laboratory, tim.scheibe@pnnl.gov

${ }^{2}$ See https://sbrsfa.pnnl.gov

River corridors are critical components of watersheds. Hydrologic exchange flows (HEFs) between river channels and subsurface environments have key functions in river corridors including stimulating biogeochemical reactions that support aquatic ecosystems and improve water quality, modulating water temperatures, and regulating nutrient concentrations. These complex systems offer many opportunities to apply new and emerging observational and modeling frameworks to gain understanding of their function, which will in turn support sustainable management of these critical ecosystems.

We present several examples focused on the generation and analysis of large datasets through a combination of observations, multiscale process-based numerical models, and data-driven analyses. We integrated bathymetric data with hydrodynamic simulations to develop a machine-guided hydromorphologic classification scheme that serves as a construct to transfer knowledge from high-resolution mechanistic models of HEFs and subsurface residence time distributions (RTDs) to large-scale reduced-order models of river corridor processes. A coupled 3D surface-subsurface model of hydrologic exchange was developed to simulate HEFs and RTDs with high spatial and temporal resolution in a $10-\mathrm{km}$ river reach with transient river discharge over a three-year period. Model outputs have been analyzed using advanced machine learning methods to evaluate the impacts of multiple environmental factors. We used these models to guide deployment of field sensors, and in turn are using field observations of HEFs, linked to geophysical observations, to test and refine our models. High-throughput analysis of organic matter chemistry and microbial community composition and function is enabling new modeling pipelines that incorporate molecular-scale information into reactive transport models, providing new and improved understanding of hydro-biogeochemical processes in dynamic rivers. Our data and models are intentionally developed within an open science framework, and we are engaging with the scientific community to tackle long-standing challenges by integrating large datasets and multiscale numerical models. 